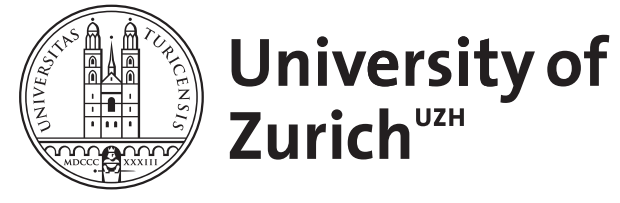

Proposal for a new radiological index to determine skull conformation in the $\operatorname{dog}$

Koch, Daniel A ; Wiestner, Thomas ; Balli, A ; Montavon, P M ; Michel, E ; Scharf, G ; Arnold, Susi

DOI: https://doi.org/10.1024/0036-7281/a000331

Posted at the Zurich Open Repository and Archive, University of Zurich ZORA URL: https://doi.org/10.5167/uzh-73471

Journal Article

Accepted Version

Originally published at:

Koch, Daniel A; Wiestner, Thomas; Balli, A; Montavon, P M; Michel, E; Scharf, G; Arnold, Susi (2012). Proposal for a new radiological index to determine skull conformation in the dog. Schweizer Archiv für Tierheilkunde, 154(5):217-220.

DOI: https://doi.org/10.1024/0036-7281/a000331 


\title{
Proposal for a new radiological index to determine skull conformation in the dog
}

\author{
D.A. Koch ${ }^{1}$, T. Wiestner ${ }^{2}$, A. Balli ${ }^{3}$, P.M. Montavon ${ }^{1}$, E. Michel ${ }^{3}$, G. Scharf ${ }^{4}$, S. Arnold $^{3}$ \\ ${ }^{1}$ Clinic for Small Animal Surgery, ${ }^{2}$ Equine Clinic, ${ }^{3}$ Clinic for Small Animal Reproduction and \\ ${ }^{4}$ Division for Diagnostical Imaging, University Zurich
}

\begin{abstract}
Measurements of the canine skull are of main interest in brachycephalic dogs. They are suspected to have diseases associated with the shortened nose, which are summarized under the term brachycephalic syndrome. The relative length of the nose has become an important feature in breeding and research, although a definitive correlation to the brachycephalic syndrome still has not been proven (Knecht, 1979; Harvey, 1982; Aron und Crowe, 1985; Koch et al., 2003). Classifications of skull types can be made by two methods. The traditional phenotypic assignment into brachycephalic, mesaticephalic and dolichocephalic is based on the assumption, that dogs of a specific breed have more or less uniform skull shapes. However, alterations due to breeding and differences between individual dogs of the same breed must lead to modifications. Therefore and for a more subtle classification, skull measurements were introduced (Brehm et al., 1985; Evans, 1993; Regodon et al., 1993),
\end{abstract}

The existing indexation methods from Evans (1993) and Brehm et al. (1985) are based on cadaver studies. They classify brachycephalic dogs as having a cranial length to facial length ratio (LL-index) of $>1.60$ or a skull length to skull width ratio (LW-index) of <1.44. However, the authors also mentioned the considerable range of those indices within a breed. Regodon et al. (1993) were the first to propose a classification based on radiographs of canine skulls with the craniofacial angle (CFA) as a criterion. The CFA is defined by a line along the base of the skull, and another line along the hard palate. The CFA of 10 dogs from 5 breeds were retrieved and it was found that the ranges clearly overlapped. The existing indices have their limitations for clinical use, either being based on cadavers (LLindex, LW-index) or not resulting in meaningful assignments of the individual skull measurement to the phenotypic appearance of the respective breed (CFA). We therefore propose a possible new skull index (S-index), which can be easily derived from radiographs of living animals and should reflect the phenotype of each individual. Such an index has also the potential to be linked to airstream parameters
(Wiestner et al., 2007) and to the clinical manifestation of respiratory problems of each individual. It offers an insight to different skull shapes between dogs of the same breed. We also propose a boundary value, which should have the potential to classify between brachycephalic and nonbrachycephalic skull shapes.

The new S-index is an approximation to the LL-index, retrieved form cadavers by Brehm et al. (1985). The index however was taken from radiographs in dorsoventral direction (Fig. 1). It is calculated by the length of the facial skull divided by the length of the cerebrum. In this context the length of the facial skull was defined as the distance from the rostral border of the incisal bone to the rostral border of the cavum cranii. The length of the cerebrum was measured from the rostral border of the cavum cranii to the caudal outline of the occipital bone, beginning at the dorsal border of the great foramen. The S-index was compared to the LW index and the CFA on 57 dogs of different breed, sex, age and size. They were presented to the University of Zurich for planned surgical procedures (mainly neutering). The owners agreed to the procedures necessary for this study. From all dogs, radiographs of the skull in dorsoventral (hard palate parallel to the table, Fig. 1) and laterolateral direction (hard palate perpendicular to the table, Fig. 2) were taken. The S- and LW-index (dorsoventral views) and CFA (laterolateral views) were determined (Tab. 1).

A boundary value (BV) for the S-index was evaluated which allows for a separation of the brachycephalic and the non-brachycephalic breeds by the following method. First, the dogs in the present study were classified on the basis of the established separating BV for the LW-index (Brehm et al., 1985). Secondly, the $\mathrm{BV}$ of $\mathrm{S}$-index was determined such that the highest score in coincidence of classification occurred for both, the brachycephalic and non-brachycephalic breeds . In this manner, it was found that all dogs having an S-index equal or below the BV of 1.25 are considered to have a brachycephalic skull shape (Tab. 1). In parallel the respective CFA were listed. 


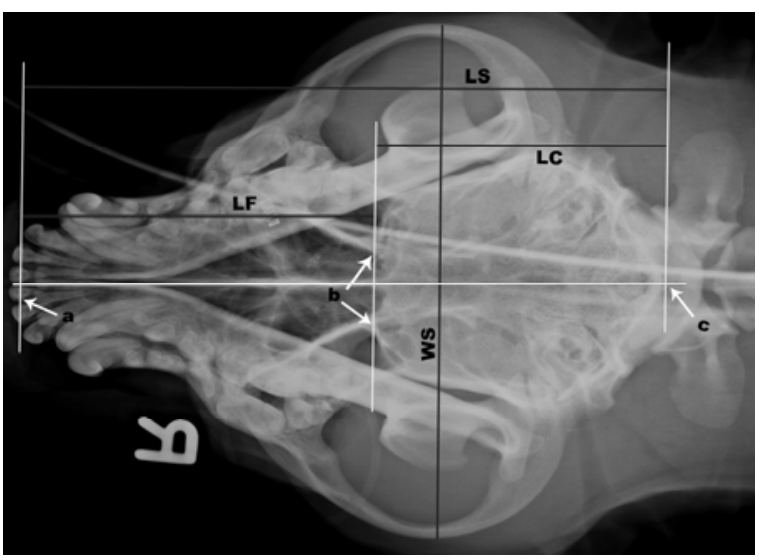

Figure 1: Dorsoventral radiograph of a canine skull with auxiliary lines and fixed points for the determination of the S-index (length of facial skull to cerebrum) and of the LW-index (length of skull to width of skull) according to Brehm et al. (1985). (LS = length of the skull; LC = length of the cerebrum; LF = length of the facial skull; WS = width of the skull; $a=$ rostral border of the incisal bone; $\mathrm{b}=$ rostral border of the cavum cranii; $\mathrm{c}=$ caudal contour of the occipital bone)

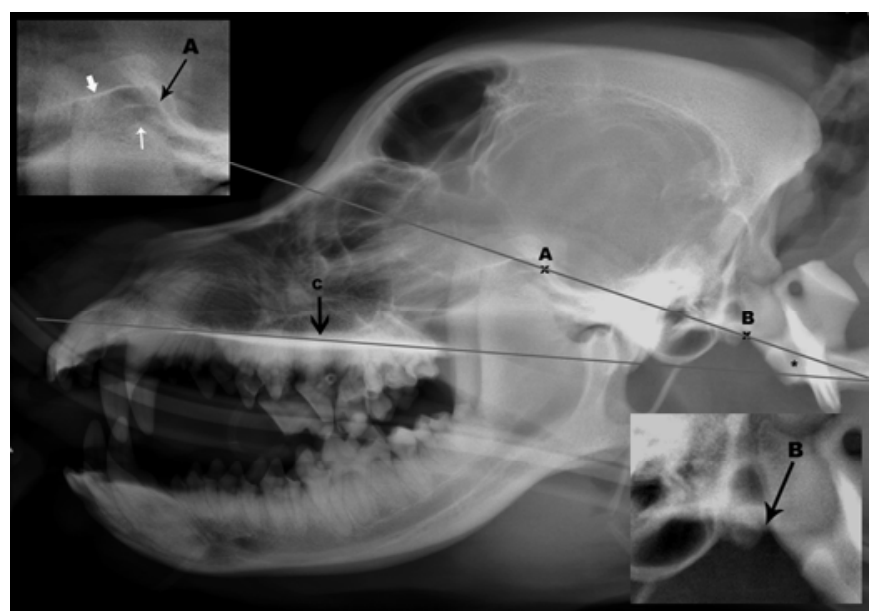

Figure 2: Laterolateral radiograph of a canine skull with auxiliary lines and fixed points for the determination of the craniofacial angle according to Regodon et al. (1993). The CFA (asterisk) is built by the prolongation of the hard palate (c) and a line connecting the sulcus chiasmatis (caudal border if the optical canal on the floor of the skull) with the caudoventral border of the occipital bone. This line is defined by two points A and B. Point A (upper left inset) is defined as the caudal edge of the middle of three radioopaque lines craniodorsal to the temporomandibular joint, representing the ventral border of the optic canal (fat white arrow), the dorsal cortex of the presphenoidal bone (thin black arrow, A) and the ventral border of the orbital fissure (thin white arrow). Point B is set, where the caudoventral contour of the occipital bone joins the contour of the occipital condyles (lower right inset).

\section{Discussion}

The proposed S-index calculated as length of the facial skull / length of the cerebrum reflects a length to length ratio. This corresponds to the definition of brachycephaly, which describes a shortening of the skull. The S-index seems to be quite equivalent to the LL-index evaluated by Brehm et al. (1985), which was determined on cadaveric skulls, using the nasal bone as a rostral border of the cerebrum. The S-index may be even more accurate, as the rostral border of the cavum cranii is clearly recognisable in radiographs and the extent of the neighbouring nasal area can be determined precisely. In cadaver skulls, the rostral border of the cavum cranii is however not visible. That is the reason why in anatomical studies (Brehm et al., 1985; Evans, 1993) the nasal bone was chosen as the rostral border. In the clinical setting, and with regard to the respiratory tract, this landmark less precisely reflects the real proportions.

The anatomic landmarks were easy to identify on all radiographs and the various lengths could be determined without problems. From these measurements, the indices were calculated as objective characteristics. Taking radiographs from living animals always carries the problem of false positioning and aberration errors. The 'length to width' indices however are more susceptible to errors than the 'length to length' indices, because the skull must be adjusted in two planes instead of only one. The S-index was compared to the LW index and the CFA in order to find a BV separating brachycephalic dogs from non-brachycephalic dogs. The determined $\mathrm{BV}$ of 1.25 for the S-index well meets this criterion, as all breeds historically known as brachycephalic had an a S-index of 1.25 or lower. Breeds belonging to this group are the pug, French bulldog, English bulldog, Cavalier King Charles spaniel, Norwich terrier, boxer, Staffordshire bullterrier and Bordeaux mastiff. Many dogs belonging to these breeds are known to suffer from the brachycephalic syndrome (Koch et al., 2003; Rosaspina, 2005). It can also be seen that breeds with an S-index >1.25, such as beagles, collies, and German shepherds, are generally classified as either mesaticephalic or dolichocephalic and the brachycephalic syndrome in these breeds is clinically not observed.

The setting of any BV reveals also some limitations, as being brachycephalic is not defined by any regulation. Although the brachycephalic dogs appeared to be listed quite similarly with respect to the $\mathrm{S}$-index and the LW-index, some dogs were at 
different ranks (Tab. 1). This confirms the observation, that within the same breed, some dogs may develop the brachycephalic syndrome and others do not. Furthermore, it points out the necessity for a subtle grading system in assessing individual skull conformations. The $\mathrm{S}$-index fulfils this criterion as is clearly visible e.g. for the group of Norwich Terriers (a classical problem breed, Ruchti, 2009), where the S-index ranged from 0.75 to 1.27 (Tab. 2). The comparison of all indices also showed, that the CFA resulted in a completely different listing. Boxer, Gordon Setter and Bergamasco sheepdog would have

Table 1: S-index, LW-index and CFA of 57 dogs from 27 different breeds arranged according to increasing S-index. For most breeds only one dog was measured. For multiple dogs of the same breed, the median value of the indices and the number of dogs (n) are given. Indices of dogs/breeds classified as brachycephalic (S-index $<=1.25$ or $\mathrm{LW}$-index $<$ 1.44) are given in bolt numbers.

\begin{tabular}{|l|c|c|c|}
\hline Breed & S & LW & CFA \\
\hline Pug & $\mathbf{0 . 1 6}$ & $\mathbf{0 . 8 8}$ & 12 \\
\hline French bulldog & $\mathbf{0 . 3 5}$ & $\mathbf{0 . 8 9}$ & 14 \\
\hline English Bulldog & $\mathbf{0 . 5 1}$ & $\mathbf{0 . 9 9}$ & 14 \\
\hline Cavalier King Charles Spaniel & $\mathbf{0 . 5 2}$ & $\mathbf{1 . 2 6}$ & 10 \\
\hline Norwich terrier $(\mathrm{n}=14)$ & $\mathbf{0 . 9 3}$ & $\mathbf{1 . 3 8}$ & 22 \\
\hline Boxer $(\mathrm{n}=3)$ & $\mathbf{0 . 9 8}$ & $\mathbf{1 . 2 7}$ & 18 \\
\hline Border Terrier & $\mathbf{1 . 0 0}$ & 1.47 & 18 \\
\hline Staffordshire bullterrier & $\mathbf{1 . 2 0}$ & $\mathbf{1 . 1 6}$ & 19 \\
\hline Bordeaux mastiff & $\mathbf{1 . 2 5}$ & $\mathbf{1 . 2 1}$ & 16 \\
\hline Jack Russell Terrier & 1.32 & 1.69 & 28 \\
\hline Beagle $(\mathrm{n}=9)$ & 1.35 & 1.67 & 24 \\
\hline Eurasier & 1.39 & 1.55 & 20 \\
\hline Lagotto Romagnolo & 1.43 & 1.53 & 20 \\
\hline Bearded collie & 1.43 & 1.73 & 26 \\
\hline Dalmatian & 1.47 & 1.66 & 20 \\
\hline Golden retriever $(\mathrm{n}=3)$ & 1.59 & 1.66 & 24 \\
\hline Greater Swiss mountain dog & 1.61 & 1.56 & 20 \\
\hline Australian shepherd & 1.64 & 1.57 & 22 \\
\hline Rottweiler & 1.64 & 1.59 & 20 \\
\hline Labrador retriever $(\mathrm{n}=4)$ & 1.82 & 1.63 & 25 \\
\hline Bergamasco sheepdog & 1.82 & 1.65 & 19 \\
\hline Gordon setter & 1.85 & 1.62 & 19 \\
\hline Rhodesian ridgeback & 1.89 & 1.69 & 20 \\
\hline Greyhound & 1.92 & 2.05 & 32 \\
\hline Kuvasz & 1.96 & 1.80 & 28 \\
\hline German shepherd $(\mathrm{n}=3)$ & 1.96 & 1.85 & 24 \\
\hline Doberman & 1.96 & 1.97 & 25 \\
\hline
\end{tabular}

nearly the same CFA. Although offering a new insight into the understanding of the brachycephaly, the CFA does not carry the potential to be helpful in the clinical setting and must be discarded from further research onto the topic. Finally, the S-index might be helpful, if it can be linked to the brachycephalic syndrome. In other words, the index could have the potential to indicate, whether an individual dog might be susceptible to develop the brachycephalic syndrome or not. It could then be used for breeding purposes.

Table 2: S-index, LW-index and CFA from 14 Norwich terriers arranged according to increasing S-index. Indices of dogs/ classified as brachycephalic (S-index $<=1.25$ or LW-index < 1.44) are given in bolt numbers.

\begin{tabular}{|c|c|c|c|}
\hline Norwich terrier & $\mathbf{S}$ & LW & CFA \\
\hline 1 & $\mathbf{0 . 7 5}$ & $\mathbf{1 . 4 0}$ & 23 \\
\hline 2 & $\mathbf{0 . 7 9}$ & $\mathbf{1 . 3 8}$ & 23 \\
\hline 3 & $\mathbf{0 . 8 0}$ & $\mathbf{1 . 3 1}$ & 21 \\
\hline 4 & $\mathbf{0 . 8 3}$ & $\mathbf{1 . 3 5}$ & 21 \\
\hline 5 & $\mathbf{0 . 8 5}$ & $\mathbf{1 . 4 1}$ & 19 \\
\hline 6 & $\mathbf{0 . 8 9}$ & 1.46 & 24 \\
\hline 7 & $\mathbf{0 . 9 3}$ & $\mathbf{1 . 3 0}$ & 18 \\
\hline 8 & $\mathbf{0 . 9 3}$ & $\mathbf{1 . 3 8}$ & 20 \\
\hline 9 & $\mathbf{0 . 9 3}$ & $\mathbf{1 . 3 7}$ & 22 \\
\hline 10 & $\mathbf{0 . 9 8}$ & $\mathbf{1 . 3 8}$ & 22 \\
\hline 11 & $\mathbf{0 . 9 8}$ & 1.53 & 23 \\
\hline 12 & $\mathbf{1 . 0 0}$ & $\mathbf{1 . 4 0}$ & 20 \\
\hline 13 & $\mathbf{1 . 0 9}$ & 1.48 & 23 \\
\hline 14 & 1.27 & $\mathbf{1 . 3 8}$ & 20 \\
\hline
\end{tabular}




\section{Conclusion}

The S-index is clinically applicable and useful to assess skull conformations. It was possible to separate breeds historically known as brachycephalic from those known as non-brachycephalic. Further in-breed separation is possible and needed, when breeding efforts should result in longer noses. The S-index still has to be linked to the brachycephalic syndrome. It is speculated, that low S-indexes would indicate upper airway obstructions. The CFA has been shown to be unreliable for classifying the skulls into brachycephalic or non-brachycephalic types.

\section{References}

Aron D.N.,Crowe D.T.: Upper airway obstruction. General principles and selected conditions in the dog and cat. Vet. Clin. North. Am. Small. Anim. Pract. 1985, 15: 891-917.

Brehm H., Loeffler K.,Komeyli H.: Schädelformen beim Hund. Zbl. Vet. Med. C. Anat. Histol. Embryol. 1985, 14: 324-331.

Evans H.E.: The Skeleton. In: Millers' anatomy of the dog. Hrsg. H. E. Evans, W.B. Saunders Company, Philadelphia, 1993, 122-218.

Harvey C.E.: Upper airway obstruction surgery 8: Overview of Results. J. Am. Anim. Hosp. Assoc. 1982, 18: 567-569.

Knecht C.D.: Upper airway obstruction in brachycephalic dogs. Comp. Cont. Educ. Pract. Vet. 1979, 1: 25-31.

Koch D.A., Arnold S., Hubler M.,Montavon P.: Brachycephalic syndrome in dogs. Comp. Cont. Educ. Pract. Vet. 2003, 25: 48-55.

Regodon S., Vivo J.M., Franco A., Guillen M.T.,Robina A.: Craniofacial angle in dolicho-, mesoand brachycephalic dogs: radiological determination and application. Anat. Anz. 1993, 175: 361-3.

Rosaspina M.: Vergleichende Untersuchungen am oberen Respirationstrakt bei Norwich Terriern, brachycephalen Hunden und mesocephalen Hunden. Dissertation, Universität Zürich, 2005.

Ruchti M.: Das obere Luftweg-Syndrom beim Norwich Terrier - Beschreibung der Erkrankung und der Untersuchungsmethoden, sowie Entwicklung und Evaluierung eines zuchthygienisch nutzbaren ScoringSchemas. Dissertation, Universität Bern, 2009.

Wiestner T.S., Koch D.A., Nad N., Balli A., Roos M., Weilenmann R., Michel E.,Arnold S.: Evaluation of the repeatability of rhinomanometry and its use in assessing transnasal resistance and pressure in dogs. Am. J. Vet. Res. 2007, 68: 178-184.

\section{Corresponding author}

Daniel Koch, Dr. med. vet, Diplomate ECVS

Daniel Koch Kleintierchirurgie AG

Rhyaecker 7

CH-8253 Diessenhofen

Switzerland

Email: daniel.koch@dkoch.ch

Phone: +41526573000

\section{Sources of funding}

The study was partly funded by the Swiss Federal Veterinary Office, Berne, Switzerland

Received: 28 June 2011

Accepted: 19 January 2012 